\title{
ON CONFORMAL MAPS WITH STARLIKE IMAGES
}

\author{
P. C. FENTON
}

\section{Introduction.}

For any positive number $r$, let $\Delta(O, r)$ be the open disc with centre $O$ and radius $r$. If $f$ is a starlike univalent function; which, for the purpose of this paper, is to say that $f$ is a regular univalent function defined on $\Delta(O, 1)$ for which both $f(0)=0$ and $f(\Delta(O, 1))$ is a set starlike with respect to $O$; then, according to a classical result of Study [5], the set $f(\Delta(O, r))$ is also starlike with respect to $O$ for any positive number $r$ less than one. Analogously, if $f$ is a convex univalent function, by which will be meant that $f$ is regular and univalent on $\Delta(O, 1)$ and $f(\Delta(O, 1))$ is a convex set, then $f(\Delta(O, r))$ is convex for any positive number $r$ less than one, a result once again due to Study [5].

Pommerenke [4] and Heins [2] have given, independently, a complete answer to a question suggested by this latter result of Study, namely: what property characterizes the sets in the disc which are always mapped onto convex sets by convex univalent functions? In part the aim of this paper is to show that the intimate connection existing between convex and starlike univalent functions, together with the result of Pommerenke and Heins, which will be stated below, is a key to the characterization of the subsets of the unit disc which are always mapped onto sets starlike with respect to $O$ by starlike univalent functions, and to describe this characterization; the other partial aim is, following Heins (see [3]), to establish 'a theorem concerning the representation of such subsets.

Before stating the theorem of Pommerenke and Heins we shall have need to introduce the notion of $D$-convexity (the name is due to Heins) which may be formulated as follows. Given two distinct points $a$ and $b$ in the open unit disc there are exactly two circles $C_{1}$ and $C_{2}$, called oricycles, which lie in the closure of $\Delta(O, 1)$, pass through both $a$ and $b$ and have a tangent in common with the unit circle. We denote by $D(a, b)$ the intersection of the two closed discs which have $C_{1}$ and $C_{2}$ as frontiers. A subset $E$ of $\Delta(O, 1)$ is then said to be $D$-convex provided that $D(a, b)$ is contained in $E$ whenever $a$ and $b$ are distinct points in $E$. The theorem of Pommerenke and Heins is: 
Suppose that $E$ is a subset of $\Delta(O, 1)$. Then $f(E)$ is a convex set for every convex univalent function $f$ if and only if $E$ is $D$-convex.

The statement of the first of the theorems to be proved here requires a similar introductory notion which, for the sake of uniformity, will be called $D$ starlikeness. A set $E$ contained in $\Delta(O, 1)$ will be called $D$-starlike if, given any non-zero point $z_{0}$ in $E$, the set

$$
\begin{aligned}
D\left(z_{0}\right)= & \left\{z: z=t z_{0} e^{i \theta}\right. \text { and } \\
& \left.|\theta| \leqq \theta_{0}\left(|z|,\left|z_{0}\right|\right)=2 \operatorname{Ar} \sin \left(\frac{\left|z_{0}\right|-|z|}{1-\left|z z_{0}\right|}\right) \text { and } 0 \leqq t \leqq 1\right\}
\end{aligned}
$$

is contained in $E$. Our result is then

THEOREM 1. Let $E$ be a subset of $\Delta(O, 1)$. Then $f(E)$ is starlike with respect to the origin for every starlike univalent $f$ if and only if $E$ is $D$-starlike.

Now it happens that the set $D\left(z_{0}\right)$ of $(1.1)$ is not itself $D$-starlike for any nonzero number $z_{0}$ in $\Delta(O, 1)$. On the way to obtaining a representation of a general $D$-starlike set, it is helpful to know what is the smallest $D$-starlike set containing $z_{0}$ and to this end we make the following definition.

Let $z_{0}$ be a non-zero number, of modulus $r_{0}$ less than one. For $t$ in the interval $\left[0, r_{0}\right]$, let $R\left(r_{0}\right)$ be the solution, if it exists, of the equation

$$
\log \left\{\frac{1+r_{0}}{1-r_{0}} \cdot \frac{1-t}{1+t}\right\}=\pi ;
$$

otherwise, let $R\left(r_{0}\right)=0$. For $r$ in the interval $\left[R\left(r_{0}\right), r_{0}\right]$, we define $\psi\left(r, r_{0}\right)$ by

$$
\psi\left(r, r_{0}\right)=\log \left\{\frac{1+r_{0}}{1-r_{0}} \cdot \frac{1-r}{1+r}\right\}
$$

and we define a set $E\left(z_{0}\right)$ by

$$
\begin{aligned}
E\left(z_{0}\right)=\left\{z_{0}\right\} \cup\left\{z: z=t z_{0} e^{i \theta}\right. \text { and } \\
\left.\qquad|\theta|<\psi\left(t\left|z_{0}\right|,\left|z_{0}\right|\right) \text { and } \frac{R\left(\left|z_{0}\right|\right)}{\left|z_{0}\right|} \leqq t<1\right\} \cup\left\{|z|<R\left(\left|z_{0}\right|\right)\right\}
\end{aligned}
$$

Our second theorem, together with an immediate corollary which provides the representation we are seeking, is then

THeOREM 2. For any non-zero number $z_{0}$ in $\Delta(0,1), E\left(z_{0}\right)$ is the smallest $D$ starlike set containing $z_{0}$. 
Corollary. Every D-starlike set in $\Delta(O, 1)$ is of the form $\bigcup_{z \in S} E(z)$, where $S$ is a subset of $\Delta(O, 1)$; conversely, given any non-empty subset $S$ of $\Delta(O, 1)$, $\bigcup_{z \in S} E(z)$ is D-starlike.

\section{Preliminaries.}

The connection between starlike and convex univalent functions alluded to previously may be gleaned from the following two results (see $[1, \mathrm{pp} .166-$ 167]):

(i) A function $f(z)$, regular in $\Delta(0,1)$ and satisfying both $f(0)=0$ and $f^{\prime}(0)$ $=1$, is starlike univalent if and only if

$$
\operatorname{Re}\left\{\frac{z f^{\prime}(z)}{f(z)}\right\} \geqq 0 \quad \text { for } z \in \Delta(O, 1) .
$$

(ii) A function $f(z)$, regular in $\Delta(O, 1)$ and satisfying both $f(0)=0$ and $f^{\prime}(0)=1$, is convex univalent if and only if

$$
\operatorname{Re}\left\{\frac{z f^{\prime \prime}(z)}{f^{\prime}(z)}\right\} \geqq-1 \quad \text { for } z \in \Delta(O, 1) .
$$

Using (i) and (ii), we readily see that, if $f$ is starlike univalent, then

$$
g(z)=\int_{0}^{z} \frac{f(\zeta)}{\zeta} d \zeta \quad \text { for } z \in \Delta(O, 1)
$$

is convex univalent. For if $f(z)$ is starlike univalent, then $f(0)=0, f^{\prime}(0)=c, c \neq 0$, so with $f_{1}(z)=c^{-1} f(z)$, we have, by (i), $\operatorname{Re}\left\{z f_{1}^{\prime}(z) f_{1}(z)^{-1}\right\} \geqq 0$ for $z$ in $\Delta(0,1)$. With $g_{1}(z)$ defined by

$$
g_{1}(z)=\int_{0}^{z} \frac{f_{1}(\zeta)}{\zeta} d \zeta, \quad z \in \Delta(O, 1),
$$

if follows that $g_{1}(0)=0, g_{1}^{\prime}(0)=1$ and

$$
\operatorname{Re}\left\{z g_{1}^{\prime \prime}(z) g_{1}^{\prime}(z)^{-1}\right\} \geqq-1, \quad \text { for } z \in \Delta(O, 1),
$$

and so, by (ii), $g_{1}(z)$ is convex univalent. Thus, by our definition, so is $g(z)$ $=c g_{1}(z)$.

\section{Proof of Theorem 1: Sufficiency.}

We begin the proof of Theorem 1, considering first the sufficiency of the $D$. starlikeness condition.

Suppose that $E$ is a $D$-starlike set contained in $\Delta(O, 1)$, and let $z_{0}$ be any fixed non-zero point in $E$. Let $f(z)$ be a starlike univalent function and let $g(z)$ be 
defined by (2.1), so that $g$ is convex univalent. Let $\Gamma: z=\zeta(t), 0 \leqq t \leqq 1$, be the curve in $\Delta(O, 1)$ defined by $f(\zeta(t))=t f\left(z_{0}\right), 0 \leqq t \leqq 1$, which certainly exists since $f$ is starlike; we aim to show that $\Gamma$ is contained in $E$.

Let us first note that, since $f$ is starlike univalent,

$$
\frac{d}{d t} \log |\zeta(t)|>0 \quad \text { for } 0<t \leqq 1,
$$

so that any circle about the origin intersects $\Gamma$ at most once. For we know that $\operatorname{Re}\left(z f^{\prime}(z) f(z)^{-1}\right) \geqq 0$ and consequently, since $f(\zeta(t))=t f\left(z_{0}\right)$,

$$
\frac{d}{d t} \log |\zeta(t)|=\operatorname{Re}\left\{\frac{\zeta^{\prime}(t)}{\zeta(t)}\right\}=\frac{1}{t} \operatorname{Re}\left\{\frac{f(\zeta(t))}{\zeta(t) f^{\prime}(\zeta(t))}\right\}>0 .
$$

Let $r$ be a fixed positive number less than $r_{0}=\left|z_{0}\right|$ and let $\tau$ be the number for which $|\zeta(\tau)|=r, 0<\tau<1$. Then

$$
\arg \left\{\frac{\zeta(\tau)}{z_{0}}\right\}=\arg \left\{\frac{f\left(z_{0}\right)}{z_{0}} \cdot \frac{\zeta(\tau)}{f(\zeta(\tau))}\right\}=\arg \left\{\frac{g^{\prime}\left(z_{0}\right)}{g^{\prime}(\zeta(\tau))}\right\},
$$

where "arg" denotes the many-valued function. (We denote the principal value by "Arg".) Now, let $\gamma: z=\xi(t), 0 \leqq t \leqq 1, \xi(0)=z_{0}, \xi(1)=\zeta(\tau)$, be the curve in $\Delta(O, 1)$ joining $z_{0}$ to $\zeta(\tau)$ which is mapped by $g$ onto the line segment joining $g\left(z_{0}\right)$ to $g(\zeta(\tau))$. Then

$$
\begin{aligned}
\arg g^{\prime}\left(z_{0}\right) & =\arg \left\{\lim _{t \rightarrow 0+} \frac{g(\xi(t))-g\left(z_{0}\right)}{\xi(t)-\xi(0)}\right\} \\
& =\arg \left\{\frac{g(\zeta(\tau))-g\left(z_{0}\right)}{\xi^{\prime}(0)}\right\}
\end{aligned}
$$

since $\arg \left(g(\xi(t))-g\left(z_{0}\right)\right)$ is the same for all $t, 0<t \leqq 1$; and similarly

$$
\begin{aligned}
\arg g^{\prime}(\zeta(\tau)) & =\arg \left\{\lim _{t \rightarrow 1-} \frac{g(\zeta(\tau))-g(\xi(t))}{\xi(1)-\xi(t)}\right\} \\
& =\arg \left\{\frac{g(\zeta(\tau))-g\left(z_{0}\right)}{\xi^{\prime}(1)}\right\} .
\end{aligned}
$$

Taking (3.1), (3.2) and (3.3) together, we obtain

$$
\arg \left\{\frac{\zeta(\tau)}{z_{0}}\right\}=\arg \left\{\frac{\xi^{\prime}(1)}{\xi^{\prime}(0)}\right\},
$$

and we set about to estimate the right hand side of (3.4). As one case we suppose that

$$
0 \leqq \operatorname{Arg}\left\{\frac{\zeta(\tau)}{z_{0}}\right\} \leqq \pi .
$$


(The remaining case, of $-\pi<\operatorname{Arg}\left\{\zeta(\tau) / z_{0}\right\}<0$, admits of a precisely similar treatment.) From the result of Pommerenke and Heins we know that $\gamma$ is contained within the intersection $D\left(\zeta(\tau), z_{0}\right)$ of the closed discs the frontiers of which are the oricycles $C_{1}$ and $C_{2}$ passing through $\zeta(\tau)$ and $z_{0}$. Of these two oricycles, one of them, say $C_{1}$, is such that the argument of the anticlockwise tangent vector increases as we pass from $z_{0}$ along $C_{1} \cap D\left(\zeta(\tau), z_{0}\right)$ to $\zeta(\tau)$. Let the centre of this oricycle be $O_{1}$ and let $\theta_{1}$ be the angle subtended by $C_{1} \cap D\left(\zeta(\tau), z_{0}\right)$ at $O_{1}$. Let the centre of $C_{2}$ be $O_{2}$ and let $\theta_{2}$ be the angle subtended by $C_{2} \cap D\left(\zeta(\tau), z_{0}\right)$ at $O_{2}$. If $0<\operatorname{Arg}\left\{\zeta(\tau) / z_{0}\right\}<\pi$, then $C_{1}$ is the circle which touches $|z|=1$ at a point within the half-plane which contains $O$ and has $\zeta(\tau)$ and $z_{0}$ on its frontier, and consequently $0<\theta_{1}<\pi$; also $0<\theta_{2}<2 \pi$ and $0<\theta_{1}+\theta_{2}<2 \pi$. These inequalities obviously continue to hold in the extreme cases, and therefore hold for $0 \leqq \operatorname{Arg}\left\{\zeta(\tau) / z_{0}\right\} \leqq \pi$.

Let $w_{1}\left(z_{0}\right)$ be a complex number with the direction of the anticlockwise tangent vector of $C_{1}$ at $z_{0}$, and let $w_{1}(\zeta(\tau))$ be a complex number with its direction at $\zeta(\tau)$. Similarly, let $w_{2}\left(z_{0}\right)$ be a complex number with the direction of the clockwise tangent vector of $C_{2}$ at $z_{0}$, and let $w_{2}(\zeta(\tau))$ be a complex number with its direction at $\zeta(\tau)$. Elementary geometry reveals that both

$$
\operatorname{sgn}\left\{\frac{w_{1}(\zeta(\tau))}{w_{1}\left(z_{0}\right)}\right\}=e^{i \theta_{1}}, \quad \operatorname{sgn}\left\{\frac{w_{2}(\zeta(\tau))}{w_{2}\left(z_{0}\right)}\right\}=e^{-i \theta_{2}},
$$

and we therefore deduce that

$$
\operatorname{sgn}\left\{\frac{\xi^{\prime}(1)}{\xi^{\prime}(0)}\right\}=e^{i \varphi}, \quad \text { where }-\theta_{2} \leqq \varphi \leqq \theta_{1} .
$$

Let us review what we have obtained. The numbers $\theta_{1}$ and $\theta_{2}$ of equation (3.5) are uniquely determined by $\zeta(\tau)$ and $z_{0}$, which means, since we are considering $z_{0}$ and $|\zeta(\tau)|$ fixed and restricting ourselves to those functions $f$ for which

$$
0 \leqq \operatorname{Arg}\left\{\frac{\zeta(\tau)}{z_{0}}\right\}=\theta \leqq \pi,
$$

that $\theta_{1}$ and $\theta_{2}$ depend only on $\theta$. Thus equation (3.5) establishes the possible range of values of $\varphi$ for each $\theta$, and the problem now is to determine those values of $\theta$ for which (3.4) (which may be rewritten as: $\theta=\varphi$ ) and (3.5) are compatible.

\section{A Lemma.}

Let $r_{0}$ be a fixed positive number less than one and let $\zeta=r e^{i \theta}$, where $r$ is a fixed positive number less than $r_{0}$ and $0 \leqq \theta \leqq \pi$. Let $C(\zeta)$ be the oricycle passing through $r_{0}$ and $\zeta$ which touches the circle $|z|=1$ at a point in the half plane which 
contains $O$ and has $\zeta$ and $r_{0}$ as boundary points. (In case $\theta=0$ or $\pi, C(\zeta)$ is the oricycle with centre in the lower half plane.) Let $\varphi(\zeta)$ be the angle subtended at its centre by the arc of $C(\zeta)$, obtained by passing anticlockwise from $r_{0}$ to $\zeta$. Then

$$
\begin{array}{ll}
\varphi(\zeta)>\theta & \text { for } 0 \leqq \theta<\theta_{0}=\theta_{0}\left(r, r_{0}\right)=2 \operatorname{Arsin}\left\{\frac{r_{0}-r}{1-r r_{0}}\right\}, \\
\varphi(\zeta)=\theta & \text { for } \theta=\theta_{0}, \\
\varphi(\zeta)<\theta & \text { for } \theta_{0}<\theta \leqq \pi .
\end{array}
$$

It is certainly the case that $\varphi(r)>0$ and also that $\varphi(-r)<\pi$. Since $\varphi(\zeta)-\theta$ is a continuous function of $\theta$ for $0 \leqq \theta \leqq \pi$, the Lemma will be proved when it is shown that $\varphi(\zeta)-\theta=0$ implies that

$$
\theta=2 \operatorname{Arsin}\left\{\frac{r_{0}-r}{1-r_{0} r}\right\} .
$$

Suppose that $\varphi(\zeta)-\theta=0$ when $\theta=\theta_{0}$, for some $\theta_{0}$ satisfying $0<\theta_{0}<\pi$. Let $\zeta_{0}=\mathrm{re}^{i \theta_{0}}$ and let us consider the circle $K$ determined by $O, \zeta_{0}$ and $r_{0}$. Since $\varphi\left(\zeta_{0}\right)$ $=\theta_{0}$ it follows that the centre, say $\eta_{0}$, of $C\left(\zeta_{0}\right)$ lies on $K$. Further, an elementary geometrical argument shows that the order of the points, passing around $K$ anticlockwise from $r_{0}$ is: $r_{0}, \zeta_{0}, O, \eta_{0}$. (Since $O$ and $\eta_{0}$ both lie on the same side of the line through $\zeta_{0}$ and $r_{0}$, the only alternative is $r_{0}, \zeta_{0}, \eta_{0}, O$. This, however, is impossible: for $\eta_{0}$ must lie on the perpendicular bisector of the line segment $\left(\zeta_{0}, r_{0}\right)$, and this cuts the real axis at a positive point.) Since $\eta_{0}$ is the centre of an oricycle passing through $\zeta_{0}$ and $r_{0}$ we find, on setting $\left|\eta_{0}\right|=x$, that

$$
\left|r_{0}-\eta_{0}\right|=1-x=\left|\zeta_{0}-\eta_{0}\right| \text {. }
$$

The triangle $\left(\zeta_{0}, \eta_{0}, r_{0}\right)$ being isosceles, angle $\left(\eta_{0}, \zeta_{0}, r_{0}\right)=\frac{1}{2} \pi-\frac{1}{2} \theta_{0}$. Also, since $r_{0}$, $\zeta_{0}, O$ and $\eta_{0}$ all lie on a circle it follows that

$$
\text { angle }\left(\eta_{0}, O, r_{0}\right)=\text { angle }\left(\eta_{0}, \zeta_{0}, r_{0}\right)=\frac{1}{2} \pi-\frac{1}{2} \theta_{0},
$$

from which we conclude further that

$$
\text { angle }\left(\eta_{0}, O, \zeta_{0}\right)=\theta_{0}+\text { angle }\left(\eta_{0}, O, r_{0}\right)=\frac{1}{2} \pi+\frac{1}{2} \theta_{0} .
$$

We apply the cosine rule to the triangle $\left(\eta_{0}, O, r_{0}\right)$ and obtain, making use of (4.1) and (4.2),

$$
x^{2}+r_{0}^{2}-2 r_{0} x \operatorname{Cos}\left(\frac{1}{2} \pi-\frac{1}{2} \theta_{0}\right)=(1-x)^{2},
$$

which gives, on simplification.

$$
2 x=\frac{1-r_{0}^{2}}{1-r_{0} \operatorname{Sin} \frac{1}{2} \theta_{0}} .
$$


Likewise, on applying the cosine rule to the triangle $\left(\eta_{0}, O, \zeta_{0}\right)$ and making use of (4.1) and (4.3) we obtain

$$
2 x=\frac{1-r^{2}}{1+r \operatorname{Sin} \frac{1}{2} \theta_{0}} .
$$

Taken together, (4.4) and (4.5) imply that

$$
\operatorname{Sin} \frac{1}{2} \theta_{0}=\frac{r_{0}^{2}-r^{2}}{r\left(1-r_{0}^{2}\right)+r_{0}\left(1-r^{2}\right)}=\frac{r_{0}-r}{1-r r_{0}}
$$

and the Lemma is proved.

\section{Conclusion of the proof of sufficiency.}

We write as before $\theta=\operatorname{Arg}\left\{\zeta(\tau) / z_{0}\right\}$. If $\theta$ belongs to the interval $\left[0, \theta_{0}\left(r, r_{0}\right)\right]$, then (3.4) and (3.5) are compatible since in this range $\theta$ also belongs to the interval $\left[0, \theta_{1}\right]$, (by the Lemma) to which $\varphi$ may also belong; that is, $\theta=\varphi$ is possible. If $\theta$ was slightly larger than $\theta_{0}\left(r, r_{0}\right)$, however, we know from the Lemma that $\theta>\theta_{1}$. We know also that $\theta_{1}+\theta_{2}<2 \pi$ always and it would be, therefore, possible to choose $\theta$ satisfying $\theta_{1}<\theta<2 \pi-\theta_{2}$; but then (3.4) would imply $\theta_{1}<\varphi<2 \pi-\theta_{2}$, which contradicts (3.5). We thus conclude that $\theta$ can belong only to one or other of the intervals

$$
I_{1}\left(r, r_{0}\right)=\left[0, \theta_{0}\left(r, r_{0}\right)\right], \quad I_{2}\left(r, r_{0}\right)=\left[\theta_{0}\left(r, r_{0}\right)+\delta\left(r, r_{0}\right), \pi\right],
$$

where $\delta\left(r, r_{0}\right)$ satisfies $0<\delta\left(r, r_{0}\right) \leqq \pi-\theta_{0}\left(r, r_{0}\right)$ for $0<r<r_{0}$. The case of $-\pi<\theta$ $<0$ is dealt with in a precisely similar way and combining the two results we find that, if $|\zeta(\tau)|=r$, then $\theta=\operatorname{Arg}\left\{\zeta(\tau) / z_{0}\right\}$ must belong to one of the following intervals:

$$
\begin{array}{r}
{\left[-\theta_{0}\left(r, \dot{r}_{0}\right), \theta_{0}\left(r, r_{0}\right)\right], \quad\left[\theta_{0}\left(r, r_{0}\right)+\delta\left(r, r_{0}\right), \pi\right],} \\
\\
{\left[-\pi,-\theta_{0}\left(r, r_{0}\right)-\delta\left(r, r_{0}\right)\right] .}
\end{array}
$$

The first interval defines the region $D\left(z_{0}\right)$ of (1.1) as $r$ varies between zero and $r_{0}$, and the remaining two intervals together define a second region $F\left(z_{0}\right)$ which can have at most two points in common with $D\left(z_{0}\right)$, namely $z_{0}$ and $O$. Let us observe that, given $r_{0}, \theta$ must lie in $D\left(z_{0}\right)$ for small values of $r$. For if $r$ is small, $\theta_{2}$ (the angle introduced in Section 3 ) is less than $\pi$ no matter what the value of $\theta$, and therefore if $\theta$ belongs to $[0, \pi]$ it must also belong to $\left[0, \theta_{0}\left(r, r_{0}\right)\right]$ and if $\theta$ belongs to $(-\pi, 0)$ it must also belong to $\left[-\theta_{0}\left(r, r_{0}\right), 0\right]$. That $\Gamma$ is contained in $D\left(z_{0}\right)$ now follows at once: for $\Gamma$ is a continuous curve which lies in $D\left(z_{0}\right) \cup F\left(z_{0}\right)$, begins at $O$ and has its initial section in $D\left(z_{0}\right)$, ends at $z_{0}$ and crosses each circle about the origin at most once. Thus $\Gamma$ lies completely in $D\left(z_{0}\right)$ and so by hypothesis, in $E$, the $D$-starlike set with which we began. Since 
this is true for each non-zero $z_{0}$ in $E, f(E)$ is starlike with respect to the origin for every starlike univalent function $f$. The proof of sufficiency is thereby concluded.

\section{Proof of Theorem 1: Necessity.}

Suppose that $E$ is a set in $\Delta(O, 1), E \neq\{O\}$ or $\varnothing$, which is such that the set $f(E)$ is starlike with respect to $O$ for every starlike univalent $f$. Then $E$ is a starlike set with respect to the origin and so, since $D\left(z_{0}\right)$ is also starlike with respect to the origin, we will have proved that $D\left(z_{0}\right) \subset E$ once we have shown that $\partial D\left(z_{0}\right) \subset E$, for any non-zero $z_{0}$ in $E$.

Given a non-zero $z_{0}$ in $E$, let $\zeta=r \operatorname{sgn}\left(z_{0}\right) e^{i \theta_{0}\left(r, r_{0}\right)}$ be a boundary point of $D\left(z_{0}\right)$, where $r_{0}=\left|z_{0}\right|$ and $0<r<r_{0}$. Let $C$ be the oricycle passing through $z_{0}$ and $\zeta$ which touches the circle $|z|=1$ at a point $\eta$ within the half-plane containing $O$ and having $z_{0}$ and $\zeta$ as frontier points. For some real number $R$ and some number $\eta$ with $|\eta|=1$, then, $C$ is described by the equation

$$
\operatorname{Re}\left\{\frac{\eta+z}{\eta-z}\right\}=R \text {. }
$$

Let

$$
g(z)=\frac{\eta+z}{\eta-z},
$$

a convex univalent function, and let $f(z)=z g^{\prime}(z)$, a starlike univalent function. We consider

$$
\begin{aligned}
\operatorname{Arg}\left\{\frac{f(\zeta)}{f\left(z_{0}\right)}\right\} & =\operatorname{Arg}\left\{\frac{\zeta g^{\prime}(\zeta)}{z_{0} g^{\prime}\left(z_{0}\right)}\right\} \\
& =\operatorname{Arg}\left\{e^{i \theta_{0}\left(r, r_{0}\right)} \frac{g^{\prime}(\zeta)}{g^{\prime}\left(z_{0}\right)}\right\}
\end{aligned}
$$

It follows from the definition of $\theta_{0}\left(r, r_{0}\right)$ that the angle subtended by the minor arc $\left(\zeta, z_{0}\right)$ at the centre of $C$ is $\theta_{0}\left(r, r_{0}\right)$. From this, together with considerations similar to those leading to (3.2) and (3.3), it follows that

$$
\operatorname{Arg}\left\{\frac{g^{\prime}(\zeta)}{g^{\prime}\left(z_{0}\right)}\right\}=e^{-i \theta_{0}\left(r, r_{0}\right)}
$$

We deduce from (6.1) and (6.2) that

$$
\operatorname{Arg}\left\{\frac{f(\zeta)}{f\left(z_{0}\right)}\right\}=0 .
$$


Since $|\zeta|<\left|z_{0}\right|$, it follows that $\zeta$ lies on the curve $\Gamma: z=\zeta(t)$ defined by $f(\zeta(t))$ $=t f\left(z_{0}\right), 0 \leqq t \leqq 1$, which lies in $E$ by hypothesis. Hence $\partial \mathrm{D}\left(z_{0}\right) \subset E$ and the Theorem is completely proved.

\section{Proof of Theorem 2.}

We show first that, for any non-zero $z_{0}$ in $\Delta(O, 1), E\left(z_{0}\right)$ is $D$-starlike. We assume without loss of generality that $0<z_{0}=r_{0}<1$ and observe that, since $D(z) \supset D(t z)$ for any $t$ in $[0,1]$, it is sufficient to show that $D(z) \backslash\{z\} \subset E\left(z_{0}\right)$ for any $z$ on the boundary of $E\left(z_{0}\right)$. With this in mind, let $r$ and $r_{1}$ be positive numbers such that $R\left(r_{0}\right) \leqq r<r_{1}<r_{0}$, and let $z_{1}=r_{1} e^{i \psi\left(r_{1}, r_{0}\right)}$, where $\psi\left(r, r_{0}\right)$ is the function of (1.2). We aim to show that

$$
\Delta(r)=\psi\left(r, r_{0}\right)-\left(\theta_{0}\left(r, r_{1}\right)+\psi\left(r_{1}, r_{0}\right)\right)>0, \quad \text { for } R\left(r_{0}\right) \leqq r<r_{1} ;
$$

for, if (7.1) holds then evidently $D\left(z_{1}\right) \backslash\left\{z_{1}\right\} \subset E\left(r_{0}\right)$.

By definition,

$$
\begin{aligned}
\Delta(r) & =\log \left\{\frac{1+r_{0}}{1-r_{0}} \cdot \frac{1-r}{1+r}\right\}-\log \left\{\frac{1+r_{0}}{1-r_{0}} \cdot \frac{1-r_{1}}{1+r_{1}}\right\}-2 \operatorname{Arsin}\left\{\frac{r_{1}-r}{1-r r_{1}}\right\} \\
& =\log \left\{\frac{1+r_{1}}{1-r_{1}} \cdot \frac{1-r}{1+r}\right\}-2 \operatorname{Arsin}\left\{\frac{r_{1}-r}{1-r r_{1}}\right\},
\end{aligned}
$$

and consequently

$$
\begin{aligned}
\Delta^{\prime}(r) & =\frac{1+r}{1-r}\left\{\frac{-1}{1+r}-\frac{1-r}{(1+r)^{2}}\right\}-2 \frac{d}{d r} \operatorname{Arsin}\left\{\frac{r_{1}-r}{1-r r_{1}}\right\} \\
& =-\frac{2}{1-r^{2}}+\frac{2}{1-r r_{1}}\left\{\frac{1-r_{1}^{2}}{1-r^{2}}\right\}^{\frac{1}{2}} \\
& =\frac{-2}{\left(1-r r_{1}\right)\left(1-r^{2}\right)}\left\{\left(1-r r_{1}\right)-\left(1-r^{2}-r_{1}^{2}+r^{2} r_{1}^{2}\right)^{\frac{1}{2}}\right\} ;
\end{aligned}
$$

and this is negative, as is seen by squaring each term within the bracket. Thus, since $\Delta\left(r_{1}\right)=0$, we have $\Delta(r)>0$ for $R\left(r_{0}\right) \leqq r<r_{1}$, for any $r_{1}$ in the open interval $\left(R\left(r_{0}\right), r_{0}\right)$; similarly $\Delta(r)>0$ for $R\left(r_{0}\right)<r<r_{0} . E\left(z_{0}\right)$ is therefore $D$-starlike.

Let, now, $H$ be a $D$-starlike set containing $z_{0}$, where $z_{0}$ is an arbitrary nonzero number in $\Delta(O, 1)$. We will show that $H \supset E\left(z_{0}\right)$, which will complete the proot of Theorem 2 .

Suppose, on the contrary, that there is an angle $\varphi_{0}$, satisfying $0 \leqq \varphi_{0} \leqq \pi$, (the case of $-\pi<\varphi_{0}<0$ is approached in a precisely similar way) and a number $t_{0}, 0$ $<t_{0}<T\left(\varphi_{0},\left|z_{0}\right|\right)$, (where $t=T\left(\psi,\left|z_{0}\right|\right)$ is the inverse function of $\psi=\psi\left(t,\left|z_{0}\right|\right)$ of (1.2)) for which $t_{0} e^{i \varphi_{0}}$ does not belong to $H$. If $H \ngtr E\left(z_{0}\right)$, some such $\varphi_{0}$ must 
exist. Moreover, since $H$ is at least starlike, it follows that $z=t e^{i \varphi_{0}} \notin H$ for any $t$ $\geqq t_{0}$, in particular for $T\left(\varphi_{0},\left|z_{0}\right|\right) \geqq t \geqq t_{0}$. From the $D$-starlikeness of $H$, it follows that the points $z=T\left(\varphi_{0},\left|z_{0}\right|\right) e^{i\left(\varphi_{0}-\varphi\right)}$ are outside $H$, for any $\varphi$ satisfying

$$
0 \leqq \varphi \leqq \varphi_{1}=\log \left\{\frac{1+T\left(\varphi_{0},\left|z_{0}\right|\right)}{1-T\left(\varphi_{0},\left|z_{0}\right|\right)} \cdot \frac{1-t_{0}}{1+t_{0}}\right\} ;
$$

for $D(z) \supset\left\{t_{0} e^{i \varphi_{0}}\right\}$ for all such $z$, so if one of these $z$ were in $H$, we would have $H \supset D(z) \supset\left\{t_{0} e^{i \varphi_{0}}\right\}$. Therefore all points $z=t e^{i\left(\varphi_{0}-\varphi_{1}\right)}$, for $T\left(\varphi_{0}-\varphi_{1},\left|z_{0}\right|\right) \geqq t$ $\geqq T\left(\varphi_{0},\left|z_{0}\right|\right)$, lie outside $H$ and consequently, from the $D$-starlikeness of $H$, all points $z=T\left(\varphi_{0}-\varphi_{1},\left|z_{0}\right|\right) e^{i\left(\varphi_{0}-\varphi_{1}-\varphi\right)}$ are outside $H$, for any $\varphi$ satisfying

$$
0 \leqq \varphi \leqq \varphi_{2}=\log \left\{\frac{1+T\left(\varphi_{0}-\varphi_{1},\left|z_{0}\right|\right)}{1-T\left(\varphi_{0}-\varphi_{1},\left|z_{0}\right|\right)} \cdot \frac{1-T\left(\varphi_{0},\left|z_{0}\right|\right)}{1+T\left(\varphi_{0},\left|z_{0}\right|\right)}\right\}
$$

We are faced with two possibilities: either this process may be repeated indefinitely with $\varphi_{0}-\Sigma_{1}^{m} \varphi_{j}>0$ for every $m$; or, for some positive integer $M$,

$$
\sum_{1}^{M-1} \varphi_{j}<\varphi_{0} \leqq \sum_{1}^{M} \varphi_{j}
$$

Suppose that the first possibility holds. We readily deduce the recurrence equation

$\varphi_{k+1}=\log \left\{\frac{1+T\left(\varphi_{0}-\sum_{1}^{k} \varphi_{j},\left|z_{0}\right|\right)}{1-T\left(\varphi_{0}-\sum_{1}^{k} \varphi_{j},\left|z_{0}\right|\right)} \cdot \frac{1-T\left(\varphi_{0}-\sum_{1}^{k-1} \varphi_{j},\left|z_{0}\right|\right)}{1+T\left(\varphi_{0}-\sum_{1}^{k-1} \varphi_{j},\left|z_{0}\right|\right)}\right\}, \quad k \geqq 2$,

from which it follows that, for any positive integer $m$,

$$
\sum_{1}^{m} \varphi_{j}=\log \left\{\frac{1+T\left(\varphi_{0}-\sum_{1}^{m-1} \varphi_{j},\left|z_{0}\right|\right)}{1-T\left(\varphi_{0}-\sum_{1}^{m-1} \varphi_{j},\left|z_{0}\right|\right)} \cdot \frac{1-t_{0}}{1+t_{0}}\right\}
$$

If we set $\psi=\Sigma_{1}^{\infty} \varphi_{j}$, then $0 \leqq \psi \leqq \varphi_{0}$ and, from (7.3),

$$
\begin{aligned}
\psi & =\log \left\{\frac{1+T\left(\varphi_{0}-\psi,\left|z_{0}\right|\right)}{1-T\left(\varphi_{0}-\psi,\left|z_{0}\right|\right)} \cdot \frac{1-t_{0}}{1+t_{0}}\right\} \\
& =-\log \left\{\frac{1+\left|z_{0}\right|}{1-\left|z_{0}\right|} \cdot \frac{1-T\left(\varphi_{0}-\psi,\left|z_{0}\right|\right)}{1+T\left(\varphi_{0}-\psi,\left|z_{0}\right|\right)}\right\}+\log \left\{\frac{1+\left|z_{0}\right|}{1-\left|z_{0}\right|} \cdot \frac{1-t_{0}}{1+t_{0}}\right\} \\
& =\psi-\varphi_{0}+\log \left\{\frac{1+\left|z_{0}\right|}{1-\left|z_{0}\right|} \cdot \frac{1-t_{0}}{1+t_{0}}\right\} .
\end{aligned}
$$


We deduce that

$$
\varphi_{0}=\log \left\{\frac{1+\left|z_{0}\right|}{1-\left|z_{0}\right|} \cdot \frac{1-t_{0}}{1+t_{0}}\right\}
$$

which contradicts the hypothesis that $t_{0}<T\left(\varphi_{0},\left|z_{0}\right|\right)$. Consequently the second possibility must hold; that is, there is a positive integer $M$ for which (7.2) holds. This, however, would imply that the set

$$
\left\{z: z=T\left(\varphi_{0}-\sum_{1}^{M-1} \varphi_{j},\left|z_{0}\right|\right) \exp i\left(\varphi_{0}-\sum_{1}^{M-1} \varphi_{j}-\varphi\right) \text { and } 0 \leqq \varphi \leqq \varphi_{M}\right\}
$$

lies outside $H$, which in turn implies that $z_{0}$ lies outside $H$, a contradiction. Theorem 2 is thus completely proved.

\section{REFERENCES}

1. G. M. Goluzin, Geometric Theory of Functions of a Complex Variable, Amer. Math. So Translations of Mathematical Monographs, 1969.

2. M. Heins, On a theorem of Study concerning conformal maps with convex images, Mathematical Essays Dedicated to A. J. Macintyre, Edited by Hari Shankar, Ohio University Press, Athens, Ohio, 1970.

3. M. Heins, On a theorem of Study concerning conformal maps with convex images II, Math. Scand. 32 (1973), 245-257.

4. Ch. Pommerenke, Images of convex domains under convex conformal mappings, Michigan Math. J. 9 (1962), p. 257.

5. E Study, Vorlesungen über ausgewählte Gegenstände der Geometrie, Heft 2, Konforme Abbildung einfach zusammenhängender Bereiche, B. G. Teubner, Leipzig und Berlin, 1913. 